\title{
Charging at the Steady State in EPMA, SEM and ESEM
}

\author{
J. Cazaux
}

DTI, CNRS UMR 6107,UFR Sciences, BP 1039, 51687 Reims Cedex 2, France.

Most of the specific effects observed in the e-irradiated insulators result from charges trapped below the surface. At the very early beginning of this irradiation, $t \sim 0_{+}$, the charge distribution is very similar to that deduced from standard (non-charging) calculations but, via the rearrangement of electrons and holes generated by the beam, the system evolves towards a steady state rapidly attained under the standard conditions of EPMA, SEM and ESEM. To simplify, only homogeneous and thick $(\mathrm{h} \sim 1 \mathrm{~mm})$ specimens widely illuminated by the beam are considered here.

In EPMA of ground coated specimens, $\delta$ is restricted to the conductive coating and a negative charge density, Q- $\left(\mathrm{C} / \mathrm{cm}^{2}\right) \sim-\mathrm{J}_{0}(1-\eta) \mathrm{t}\left(0_{+}\right)(1)$, starts to be established via electrons trapped in the bulk down the range of the primaries, R(Fig.1a). The induced electric field and potential functions, $\mathrm{F}_{\mathrm{Z}}$ and $\mathrm{V}(\mathrm{z})$, may be evaluated from models of negative charge distribution by solving a one dimensional Poisson equation [1]. Independently from the chosen distribution, the field is always maximum at the coating/dielectric interface where de-trapping processes start when a critical field value, $F_{C}$ is reached while the de-trapped electrons are evacuated to the ground via the coating. The increase of the electric slowing down reduces the penetration depth of the primaries from $R$ to $R_{C}$ (subscript $\mathrm{C}$ for charging). Shown in fig.1, the steady state is characterized by a depleted region, $\left(0<\mathrm{z}<\mathrm{R}_{\mathrm{C}}\right)$, submitted to a uniform critical field $\mathrm{F}_{\mathrm{C}}$ due remaining charges trapped between $\mathrm{R}_{\mathrm{C}}$ and $\mathrm{R}$ in a low field region: $|\mathrm{Q}(\infty)|\left(\sim \varepsilon \mathrm{F}_{\mathrm{C}}\right.$ with $\varepsilon$ :dielectric constant). The value of $\mathrm{F}_{\mathrm{C}}$ depends upon the energy of the trapping sites. Similarly to $\mathrm{R}, \mathrm{R}_{\mathrm{C}}$ may be expressed in a form of a power law where $\mathrm{E}_{0}{ }^{\mathrm{n}}$ is changed into $\left(\mathrm{E}_{0}-\mathrm{qF}_{\mathrm{C}} \mathrm{R}_{\mathrm{C}}\right)^{\mathrm{n}}$ (2). The main consequence is a compression of the $\phi(\rho z)$ function similar to that previously obtained [1;2] but with a less field strength (see [3] for details).

For bare insulators investigated in SEM at large $\mathrm{E}_{0}$ values, there is now a double layer system, $+\&-$, at the early beginning of the irradiation: $\mathrm{Q}_{-} \sim-\mathrm{J}_{0}(1-\eta) \mathrm{t}\left(0_{+}\right)$of thickness $\mathrm{R}$ and $\mathrm{Q}_{+} \sim \mathrm{J}_{0} \delta \mathrm{t}\left(0_{+}\right)$(3) of thickness $\mathrm{s}$ as a result of the SEE emission $\delta$ (Fig 2 left). The electric field is maximum near to $\mathrm{z} \sim \mathrm{s}$ ( between + and - charges) where recombination processes take place while the surface potential becomes more and more negative inducing a progressive external slowing down of the primaries. The steady state corresponds to $\delta_{C}+\eta_{C}=1$; it is mainly characterized by a landing energy $E\left(R_{C} \sim s\right)$ $\left(\sim \mathrm{E}_{\max }\right)$ in the 1-3 keV range[4]. $\mathrm{E}\left(\mathrm{R}_{\mathrm{C}} \sim \mathrm{s}\right)$ is independent from $\mathrm{E}_{0}$ but it may change from place to place with the local change of $s$ or of $\delta$. The surface potential $V(0)=V_{S}$ is of the form:- $\mathrm{qV}_{S}=\mathrm{E}_{0^{-}}$ $E\left(R_{C} \sim s\right)$ and it results from some remaining electrons trapped between $s$ and $R$ in a low field region.

The initial situation of ESEM is similar to that of SEM but the deposition of ions+ leads rapidly to a 3-layer system with a $2^{\mathrm{d}}$ positive (and very thin) layer on the vacuum side of the surface, $\mathrm{Q}$ (ion+). The steady state nearly corresponds to the neutrality, $\mid \mathrm{Q}_{-} \sim \mathrm{Q}_{+}+\mathrm{Q}(\mathrm{ion}+)$, when is neglected the contribution of the space charge in the gap. The potential function, $\mathrm{V}(\mathrm{z})$, takes an approximate $\mathrm{S}$ shape form similar to that expected in SEM at an energy $E_{0}$ close to $E_{02}$ [4] . Its surface value, $V(0)$, is a function of the density of negative charges trapped below the surface: more electrons are trapped in the bulk and larger is the $\mathrm{V}(0)$ value, reducing then $\delta$ in consequence (Fig.2 right). This analysis is consistent with Griffin's images where the electron trapping sites appear darker [5]. 


\section{References}

[1] J. Cazaux , X-Ray Spectrometry 25 (1996) 265

[2] O.Jbara et al, X-Ray Spectrometry 26 (1997) 291

[3] J. Cazaux, Microscopy \& Microanalysis, Special Issue: "Characterization of non-conductive ..."

[4] J. Cazaux, Eur. Phys.; J.A.P. 15 (2001) 167

[5] B.J. Griffin, Scanning 22 (2001)234

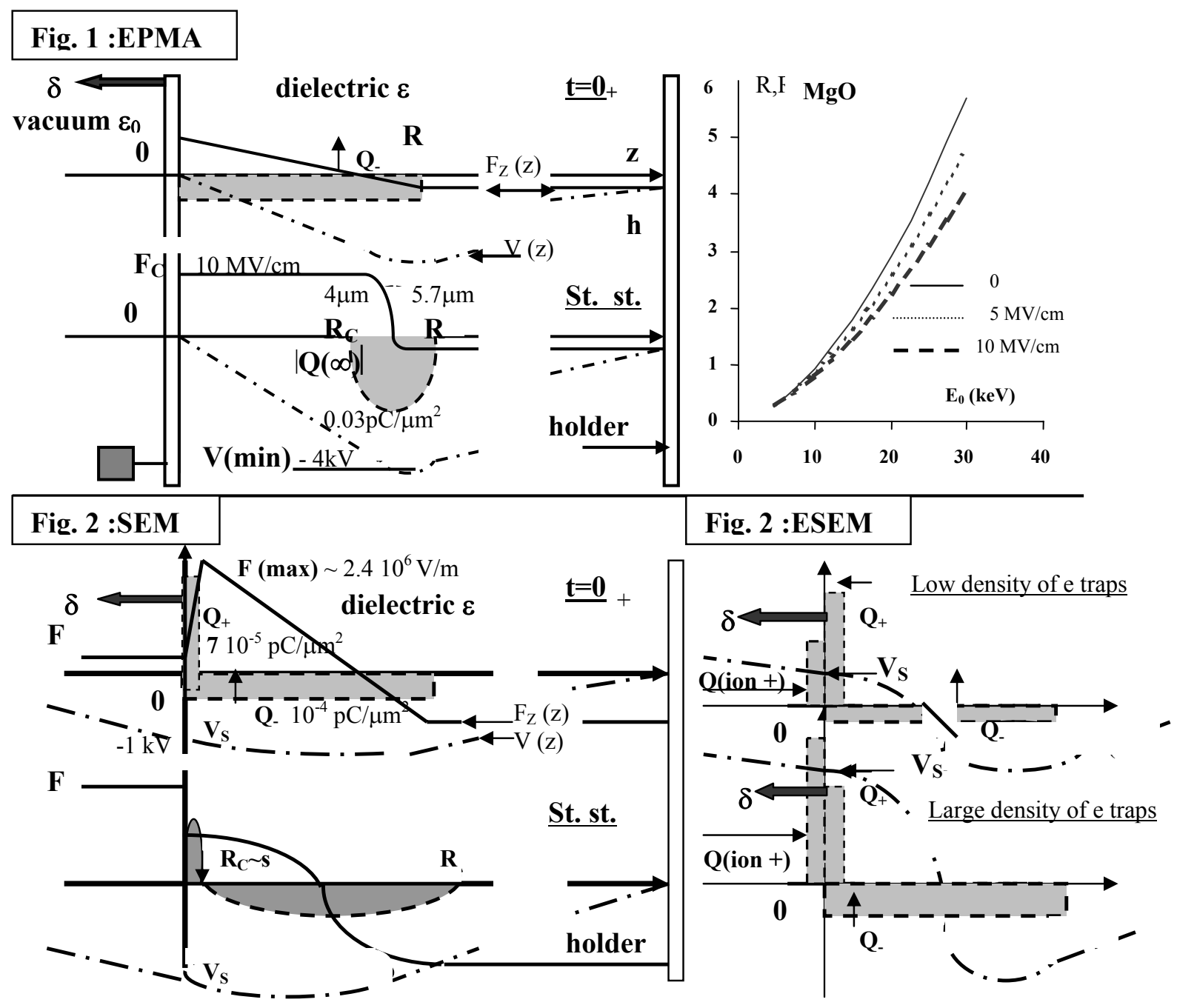

FIG.1. EPMA: $V(z)$ and $F_{Z}(z)$ functions at the early beginning of the irradiation; $t=0_{+}$(top left) and at the steady state (bottom left). For $\mathrm{MgO}$ (as an example) with $\mathrm{n}=5 / 3$ in eq.(2), influence of the field build up on the range $\mathrm{R}_{\mathrm{C}}$ of the primaries (right).

FIG. 2.Left, SEM: $V(z)$ and $F_{Z}(z)$ functions at $t=0_{+}$(top) and at the state state (bottom ).Like for fig.1, note the very low charge densities, $Q$ (that may be deduced from eqs (1)\&(3)) giving very large electric field strengths, F, and potential values, V. Right, ESEM: Expected influence of the density (top: low; bottom: large) of trapped electrons on the surface potential value, $\mathrm{V}_{\mathrm{S}}$ and on $\delta$. 